\title{
Acquired Vitamin B12 Deficiency: Cutaneous Hyperpigmentation and Hepato-Splenomegaly in Megaloblastic Anaemia / Pancytopenia Reported in a 15-Month-Old Boy
}

\author{
Faten Ahmad AlKateb ${ }^{1}$, Ahmed Mohammed AlEidan ${ }^{* 2}$, Layla Bidah AlGahtani ${ }^{2}$, Noura Muteeb AlHowiati ${ }^{3}$, \\ Aziza Fayez AlSwayyed ${ }^{4}$, Abdulaziz Khaled AlAreefy ${ }^{2}$, Mazen Mohammed AlGhofaily ${ }^{2}$ \\ ${ }^{1}$ General Paediatric Consultant, Children's Specialised Hospital, King Fahad Medical City, Riyadh, SA \\ ${ }^{2}$ Paediatric Residents, Children's Specialised Hospital, King Fahad Medical City, Riyadh, SA \\ ${ }^{3}$ Family Medicine Resident, King Fahad Medical City, Riyadh, SA \\ ${ }^{4}$ Haemato-Pathology Consultant, King Fahad Medical City, Riyadh, SA
}

Correspondence should be addressed to Ahmed Mohammed AlEidan; Ahmedaleidan@gmail.com

Received 23 August 2020;

Accepted 06 September 2020;

Published 13 September 2020

\begin{abstract}
Megaloblastic Anemia (MA) embraces various conditions with a functioning or a depressed marrow. Hence, in the light of this group of anemia, several observations were made linking it to cutaneous hyperpigmentation. Thus, in combination of both and in the presence of Pancytopenia, we report an atypical clinical image of Acquired vitamin B12 deficiency consistent with the above-mentioned description in a paediatric patient.
\end{abstract}

Keywords: Vitamin b12 deficiency, Paediatric, Anaemia, pancytopenia, Saudi Arabia.

\section{Introduction}

Cutaneous hyperpigmentation and megaloblastic anemia (MA) are tightly correlated on clinical basis, however this association is usually unrecognized among other signs of this type of anemia, which could be misleading towards other diagnoses that have a similar clinical picture. It was observed that hyper pigmented lesions confined to the Knuckle pad are much more commonly encountered than diffuse pigmentation of the palms and/or soles in such patients.

\section{Methods and materials}

We, hereby, describe and report the bone marrow (BM) changes and clinico-laboratory characteristics of a 15-month-old baby boy who underwent BMA for pancytopenia.

\section{Case report}

A 14-month-old baby boy, who was delivered to a mother diagnosed with isolated ITP during pregnancy, Iron and Vit D3 deficiency as well, at term with unremarkable neonatal period.

His complaint started at his first birthday, when a constellation of (intermittent febrile episodes, non-bilious non- projectile vomiting, diarrheal illness, and pallor which progressed to a gradual generalized skin hyperpigmentation 'Distal Interphalangeal joints, dorsal aspects of both hands and feet, and under the chin" along with fatigability, and hepato-splenomegaly) appeared in conjunction with pancytopenia, severe absolute neutropenia (Hemoglobin of $3.4 \mathrm{~g} / \mathrm{dl}$, White Blood Cell count of $2.4 * 100010 \mathrm{e} 9 / \mathrm{L}$, Absolute neutrophil count of 120 10e9/L) . Therefore, he had received Packed RBCs transfusions three times in the local hospital, aiming to elevate his hemoglobin. Looking for a definite diagnosis, he was referred to our institution (King Fahad Medical City, Riyadh, KSA) as a case of pancytopenia to rule out leukemia vs Fanconi anemia.

Parents denied any family history of a similar condition, and/or any haematological / non-haematological malignancy. Reportedly, they admitted a strong background of a likely acquired vitamin B12 deficiency among maternal adult siblings who are presently instituted on Vitamin B12 supplements. It is worth noting that the baby was exclusively breast-fed with solid food introduced at 6 months of age, nevertheless, his appetite was worsening overtime with his current complaint, and he was solely accepting the breast milk. Examination demonstrated hyper pigmented skin lesions involving both upper and lower limbs, hepatosplenomegaly ( $3 \mathrm{~cm}$ below the costal margin), with no skeletal, 
radius and/or thumb anomalies, delayed or regressed developmental milestones.

His initial work-up revealed the following:

WBC 4, 3.81*1000 10e9/L

ANC $0.32 * 100010 \mathrm{e} 9 / \mathrm{L}$

Haemoglobin $8.10 \mathrm{~g} / \mathrm{L}$

PLT $86 * 1000$ 10e9/L

Potassium $4.46 \mathrm{mmol} / \mathrm{L}$

Sodium $137 \mathrm{mmol} / \mathrm{L}$

MCV 78 fl, MCH $25.20 \mathrm{pg}$

Coagulation profile: APTT 28.2, PT 16, INR 1.37

Blood and urine cultures: Both resulted as negative.

Tumor Lysis Syndrome PANEL: Uric acid 78 umol/L, 94 umol/L Phosphate $0.95 \mathrm{mmol} / \mathrm{L}$

LDH 812 U/L (HIGH)

Folate $2.8 \mathrm{nmol} / \mathrm{L}$ (NORMAL)
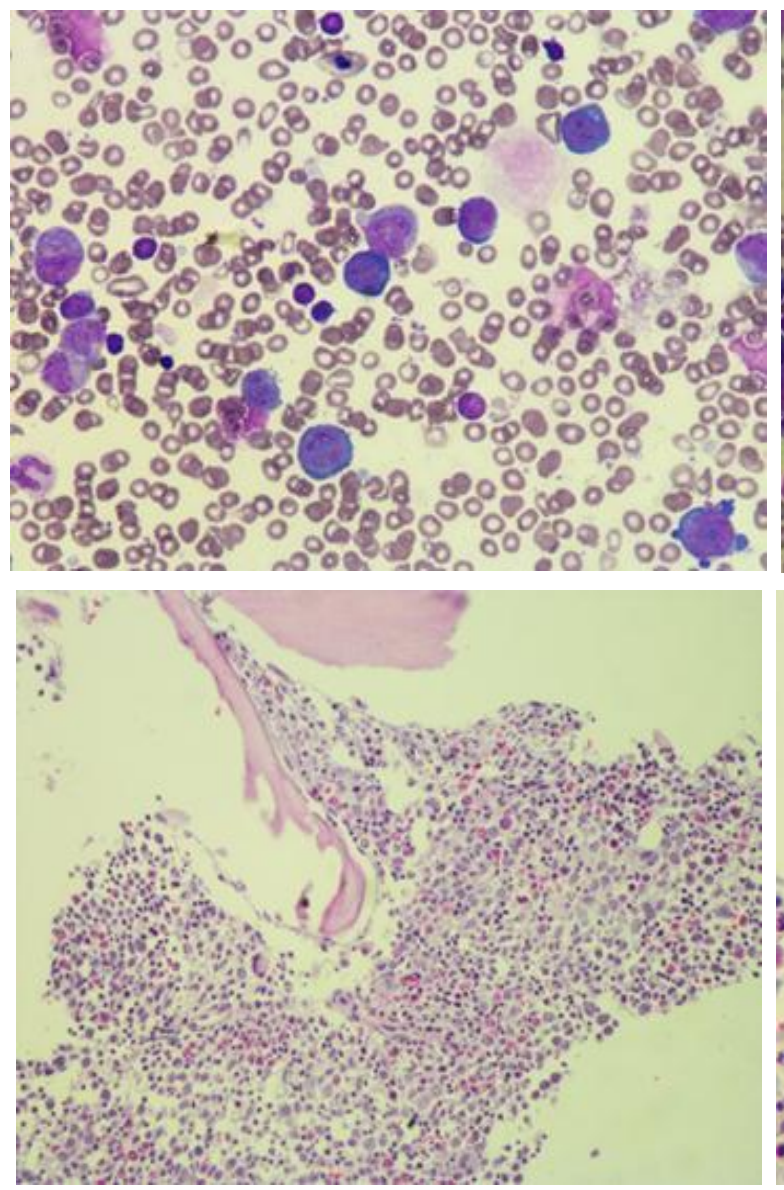

Iron 29 umol/L, Ferritin 133 ng/mL (NORMAL)

HB electropherisis (NORMAL)

CMV IgM, IgG Negative

EBV Negative

Parvo virus Negative

US abdomen: It depicted Hypo-echoic nodules on the spleen.

ACTH: $3.5 \mathrm{pmol} / \mathrm{L}(\mathrm{N})$

He was prophylactically commenced on Ampicillin-Tazobactam for his worsening pancytopenia and absolute neutropenia with a fever reported once.

Based on which, he was then subjected to a Bone Marrow Aspiration (BMA), which highlighted a normocellular bone marrow (Cellularity of 90\%) with trilineage hematopoiesis and megaloblastic features in erythroid linage, but no hyper segmented nuclei. As to rule out AML-M6 since it was of high suspicion phenotypically, a flow cytometry was sent, and leukemia was absolutely ruled out.
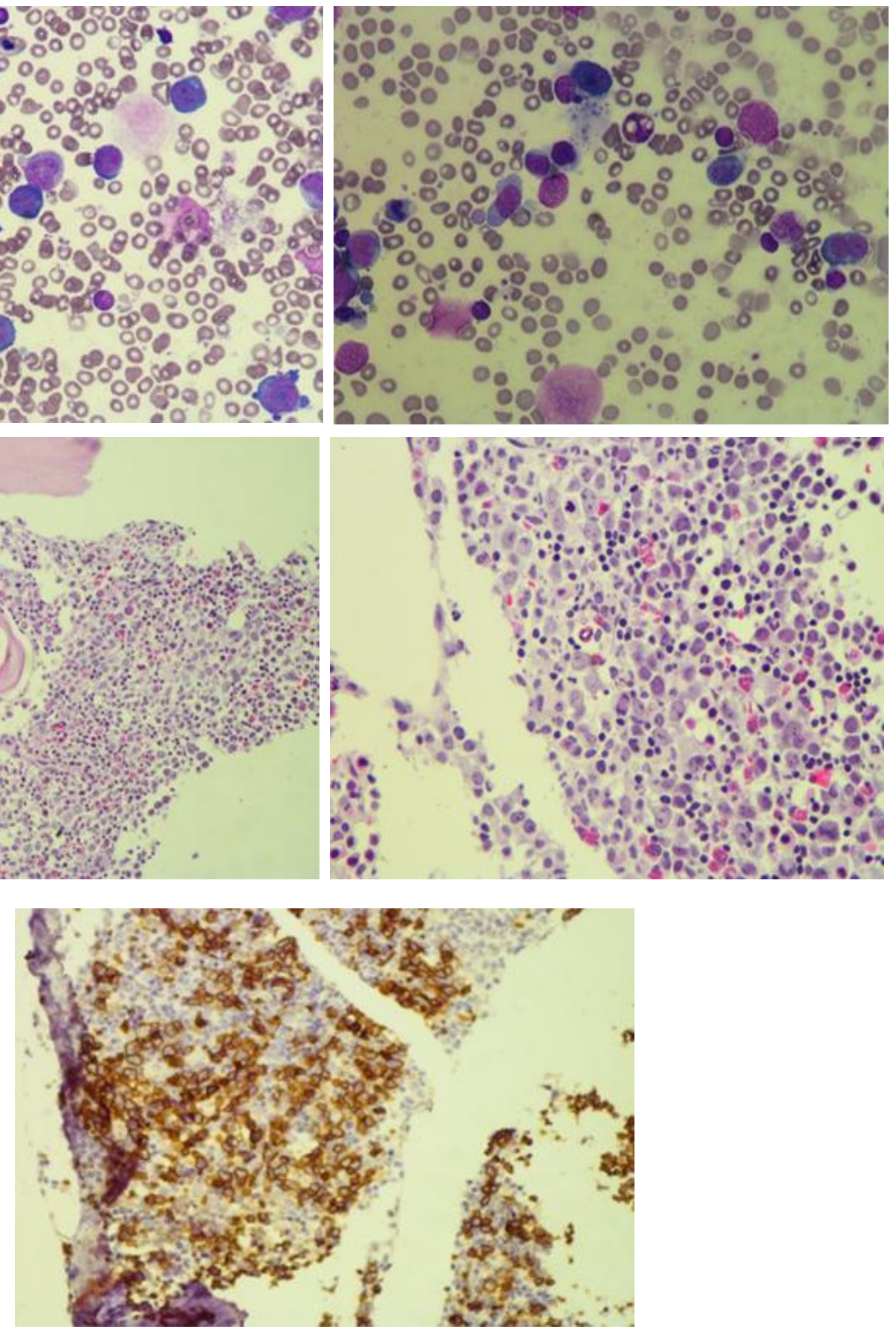

Figure 1: BMA. 
Hence we asked for vitamin b12 serum level, folate, holo transcobalamin and urine homocysteine. His Folate was 16(normal), but vitamin B12 level came up as low as (less than $111 \mathrm{pmol} / \mathrm{L}$ ) according to our normal reference ranges (138 pmol/L -652 $\mathrm{pmol} / \mathrm{L})$.

The patient was discharged on Vitamin B12 IM injections for two weeks on daily basis then to receive them once every month. A follow up appointment in 2 weeks time following the therapy was arranged and the child showed up with a remarkable improvement clinically in terms of resolved irritability, improved

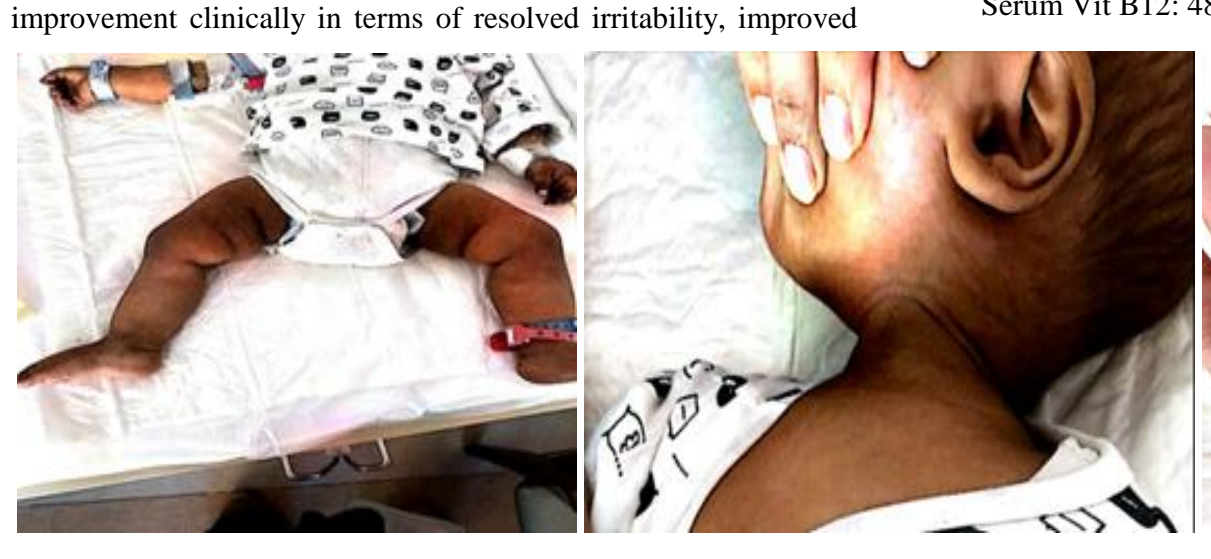

appetite, activity, and psychomotor interaction, with ability to walk independently, resolved hyper pigmentation, and investigatory as reflected on his Complete Blood Count (CBC).

His latest Lab results were as follows:

Labs

ANC $12.70 * 1000$ 10e9/L, Hb 10.80, g/L, PIT 239*1000 10e9/L, MCV $71.20 \mathrm{fl}$,

Serum Vit B12: $480 \mathrm{pmol} / \mathrm{L}$.

Figure 2: Depicted below are the child's pictures.

Keywords: Vitamin b12 deficiency, Paeditric, Anaemia, pancytopenia, Saudi Arabia.

\section{Discussion}

Vitamin B12 is a water-soluble vitamin which plays a vital role in DNA synthesis ${ }^{[1]}$. Thus, if deficient, it can cause multisystemic manifestations such as megaloblastic anaemia, neuropsychiatric, cardiovascular and gastrointestinal symptoms and much more nonspecific clinical symptomatology ${ }^{[2]}$.

Megaloblastic anemia (MA) encompasses a heterogeneous group of reversible bone marrow failure syndromes characterized by a variable degree of peripheral blood cytopenia (s) in the presence of a normo or hypercellular bone marrow. Impairment of DNA synthesis in all nucleated cells secondary to vitamin B12 (B12) and/or folate deficiency, results in nuclear-cytoplasmic asynchrony; distinctive megaloblastic changes, increased apoptosis, and ineffective hematopoiesis in the bone marrow ${ }^{[3]}$.

In a 5-year retrospective review in India by Somanath Padhi et al., Twenty-one of 25 adult cases (84\%) had MA out of which Knuckle pad hyperpigmentation (KP) was noted in $16(64 \%)$ cases; whereas 9 (36\%) had diffuse brownish black discoloration (DP) of the palms and/or soles. Cytopenia was evident in Eighteen of $25(72 \%)$ subjects. Reportedly, five cases (20\%) presented with pyrexia. Of the 17 cases where data available, eleven were B12 deficient $[<190 \mathrm{pg} / \mathrm{ml}$; eight had severe deficiency $(<100 \mathrm{pg} / \mathrm{ml})$; ref.; $190-800 \mathrm{pg} / \mathrm{ml}$ ]. In six cases where follow-up data were available, there was a significant reversal of hyperpigmentation at 12 weeks following parenteral cobalamin therapy. Whereas fever in all five cases was subsided after 24 to 72 hours following administration of parenteral Vitamin B12 therapy ${ }^{[4]}$.

Vitamin B12 deficiency was first manifested with darkening of palms and soles in 2 cases that were reported in the literature ${ }^{[5,6]}$.

At the national level, one case was reported in Jeddah, Saudi Arabia in an adult who had developed Spinal Cord Degeneration in conjunction with pancytopenia and evident vitamin B12 deficiency ${ }^{[7]}$.

\section{Conclusion}

This is the first case report in the Kingdom of Saudi Arabia that has evaluated cutaneous hyperpigmentation, hepatosplenomegaly and pancytopenia in a paediatric patient undergoing a bone marrow examination in which a lucid association with megaloblastic anemia was noted. Skin hyperpigmentation and hepatosplenomegaly were often overlooked if presented simultaneously in the light of megaloblastic anemia and cytopenia, thus our case has illustrated and intensified how these clinical signs are of paramount importance as markers in megaloblastic anemia, and Physicians should be cautious to recognize their clinical significance.

\section{IRB Approval}

Under the umbrella of KFMC IRB, this case was approved.

\section{Ethical Considerations}

A written consent was obtained and signed by the legal guardians of the baby for full disclosure while maintaining strict confidentiality in respect to the patient medical information and images under the approval of KFMC research centre ethical committee.

\section{Data Availability}

The data that support the findings of the study are available from the corresponding author upon reasonable request.

\section{Conflicts of Interest}

All authors have no example conflicts of interest to disclose.

\section{Funding Statement}

There is no funding source. 


\section{Authors' contributions}

FAA; The primary author, Reviewing the whole manuscript

AMA; Co-author, Planning the case report conception and design, reviewing relevant literature and the final writing

LBA; Co-author, Reviewing the case report scenario

NMA; Co-author, Obtaining the written and verbal consent, with the child's depicted pictures

AFA; Co-author, Reviewing the histo-pathological slides of BMA

AKA; Co-author, Reviewing discussion

MMA; Co-author, Reviewing labs

\section{Acknowledgments}

The authors would like to acknowledge Parents of the child, Children Specialized Hospital (CSH) at King Fahad Medical City (KFMC) staff for their contribution to the completion of this case report.

\section{References}

[1] P. G. Divate and R. Patanwala, "Neurological manifestations of B12 deficiency with emphasis on its aetiology," Journal of the Association of Physicians of India, vol. 62, no. 5, pp. 400-405, 2014.

[2] Stabler SP. Vitamin B12 deficiency. N Eng J Med. 2013;368:149-160. doi: 10.1056/NEJMcp1113996.
[3] Hoffbrand AV. Megaloblastic anemias. In: Fauci AS, Brunwald E, Kasper DL, et al., editors. Harrison's Principles of Internal Medicine. 17th ed. New York: McGraw-Hill; 2008. pp. 643-651.

[4] Somanath Padhi, RajLaxmi Sarangi, Anita Ramdas, Kandasamy Ravichandran, Renu G'Boy Varghese, Thomas Alexander, George Kurian, and Sudhagar Mookkappan. Cutaneous Hyperpigmentation in Megaloblastic Anemia: a Five Year Retrospective Review. Mediterr J Hematol Infect Dis. 2016; 8(1): e2016021.

[5] N. Srivastava, S. Chand, M. Bansal, K. Srivastava, and S. Singh, "Reversible hyperpigmentation as the first manifestation of dietary vitamin B12 deficiency [7]," Indian Journal of Dermatology, Venereology and Leprology, vol. 72, no. 5, pp. 389-390, 2006.

[6] K. El-Shafie, N. Samir, R. Lakhtakia et al., "Localised skin hyperpigmentation as a presenting symptom of vitamin B12 deficiency complicating chronic atrophic gastritis," Sultan Qaboos University Medical Sciences Journal, vol. 15, no. 3, pp. 420-423, 2015.

[7] Abdulhameed Alhazmi, Abdulrahman Almalki, and Safieeldin Ghazala. Skin Hyperpigmentation as the Presenting Symptom of Subacute Combined Degeneration of the Spinal Cord. Volume 2017, Article ID 7140908, 3 pages https://doi.org/10.1155/2017/7140908 